\title{
AVERAGING SEQUENCES AND ABELIAN RANK IN AMENABLE GROUPS
}

\author{
MICHAEL HOCHMAN
}

\begin{abstract}
We investigate the connection between the abelian rank of a countable amenable group and the existence of good averaging sequences (eg for the ergodic theorem).

We show that if $G$ is a group with finite abelian rank $r(G)$, then $2^{r(G)}$ is a lower bound on the constant associated to a Tempel'man sequence, and if $G$ is abelain there is a Tempel'man sequence in $G$ with this constant. On the other hand, infinite rank precludes the existence of Tempel'man sequences and forces all tempered sequences to grow super-exponentially.
\end{abstract}

\section{INTRODUCTION}

A countable group $G$ is amenable if there exists a sequence $\left\{F_{n}\right\}$ of finite subsets of $G$ with the property that for every $g \in G$,

$$
\frac{1}{\left|F_{n}\right|}\left|g F_{n} \cap F_{n}\right| \rightarrow 1
$$

(See [3] for some equivalent definitions). Such a sequence is called a Følner sequence. For example, $F_{n}=\{0, \ldots, n\} \subseteq \mathbb{Z}$ satisfy this condition.

Much of ergodic theory - ie the study of the dynamics of measure-preserving transformations - can be extended to measure-preserving actions of amenable groups. A good example of this, which explains why Følner sequences are sometimes called averaging sequences, is the mean ergodic theorem: given a measure preserving action $(g, \omega) \mapsto g \omega$ of $G$ on a probability space $(\Omega, \mathcal{F}, \mu)$, for any $\varphi \in L^{2}(\Omega, \mathcal{F}, \mu)$ and any Følner sequence $\left\{F_{n}\right\}$,

$$
\frac{1}{\left|F_{n}\right|} \sum_{g \in F_{n}} \varphi^{g} \rightarrow \mathbb{E}\left(\varphi \mid \mathcal{F}_{0}\right)
$$

in $L^{2}$. Here $\mathcal{F}_{0} \subseteq \mathcal{F}$ is the $\sigma$-algebra of $G$-invariant measurable sets, and $\varphi^{g}(x)=\varphi(g x)$. F. Riesz's proof of the mean ergodic theorem for $\mathbb{Z}$-actions carries over almost verbatim to the general case.

However, for some results - in particular for many pointwise results - it is necessary to place some restrictions on the averaging sequence $\left\{F_{n}\right\}$. One such condition is the following, which requires that the sets $F_{n}$ be invariant to some degree to translation by their own elements. As usual, we write $A B=\{a b: a \in A, b \in B\}$ and $A^{-1}=\left\{a^{-1}: a \in A\right\}$.

Definition 1.1. An increasing Følner sequence $\left\{F_{n}\right\}$ in $G$ is called a Tempel'man sequence if there is a constant $C$ such that for every $n,\left|F_{n}^{-1} F_{n}\right| \leq C\left|F_{n}\right|$

Key words and phrases. Amenable group, Følner sequence, Tempel'man sequence, tempered sequence. 
Using Tempel'man sequences in the role of averaging sequence one can prove the pointwise ergodic theorem (Birkhoff's theorem), which states that the limit (1.1) converges almost surely for $\varphi \in L^{1}$ [7], and the Shannon-McMillan-Breimann theorem [5].

Two classes of groups which possess Tempel'man sequences are the locally finite groups and the finitely generated groups with polynomial growth. For a long time no examples were known of groups without Tempel'man sequences. Recently E. Lindenstrauss [4] showed that there do exist such groups, demonstrating that the lamplighter group $L$ is one such group. This group is defined as follows: let $\mathbb{Z}$ act on $V=\oplus_{i=-\infty}^{\infty}(\mathbb{Z} / 2 \mathbb{Z})$ by coordinate shift, and set $L=\mathbb{Z} \ltimes V$.

In this paper we investigate the connection between the abelian rank of a group and the behavior of the averaging sequences it contains. Rank is defined as follows:

Definition 1.2. The abelian rank of a group $G$ is

$$
r(G)=\sup \left\{n: G \text { contains a subgroup isomorphic to } \mathbb{Z}^{n}\right\}
$$

Our first result connects abelian rank with Tempel'man sequences:

Theorem. Let $G$ be a countable group.

(1) If $r(G)<\infty$ then $2^{r(G)}$ is a lower bound on the constant associated with a Tempel'man sequence in $G$. If in addition $G$ is abelian then there exists in $G$ a Tempel'man sequence with constant $2^{r(G)}$.

(2) If $r(G)=\infty$ then $G$ has no Tempel'man sequences.

This provides many new examples of groups without Tempel'man sequences, for instance $\oplus_{i=-\infty}^{\infty} Z$.

With regard to the problem of the existence of good averaging sequences, Lindenstrauss demonstrated in [4] that tempered sequences, defined below, can serve as averaging sequences for the pointwise ergodic theorem and the SMB theorem:

Definition 1.3. A Følner sequence $\left\{F_{n}\right\}$ is tempered if there exists a constant $C$ such that $\left|\left(\cup_{i<n} F_{i}^{-1}\right) F_{n}\right| \leq C\left|F_{n}\right|$ for every $n$.

Note that every Tempel'man sequences is tempered.

Tempered sequences have the advantage that they exists in every amenable group; in fact every Følner sequence has a tempered subsequence. However tempered sequences may grow quickly. For example, every tempered sequence in the lamplighter group grows super-exponentially 4 . Fast growth implies, for instance, that in order to estimate the mean of a process from observations the size of the data set needed can grow sharply from one estimate to the next. In contrast, when calculating the mean of a $\mathbb{Z}$-process using a running average, each additional symbol of the output can be used to update the estimate.

Our second result shows that abelian rank is relevant to this question as well:

Theorem. Let $G$ be a countable group. If $r(G)=\infty$ then $G$ does not possess Tempel'man sequences, and every tempered sequence in $G$ grows super-exponentially. 
These results leave open the class of non-abelian finite rank groups, which contains groups both with and without "good" averaging sequences. We will say a little about this at the end of section 4 .

Our main tool is the observation that the Brunn-Minkowsky inequality, which bounds the volumes of sums of sets in $\mathbb{R}^{d}$, can be used to bound the sums of sufficiently invariant sets in $\mathbb{Z}^{d}$. This is developed in the next section. In section 3 we derive the results about Tempel'man sequences. Section 4 addresses the growth of tempered sequences.

Acknowledgment. This work is part of the author's M.A. thesis, conducted under the guidance of Professor Benjamin Weiss, whom I would like to thank for all his help and encouragement. I would also like to thank E. Glasner for raising some of the questions addressed here.

\section{Tempel'man SEQUENCES IN INTEGER LATTICES}

We will need the following classical theorem (see eg [1]):

Theorem. (Brunn-Minkowsky inequality) Let $A, B \subseteq \mathbb{R}^{d}$ be measurable sets such that $A+B$ is also measurable. Then, denoting the d-dimensional Lebesgue measure by $\nu$,

$$
\nu(A+B) \geq\left(\nu(A)^{1 / d}+\nu(B)^{1 / d}\right)^{d}
$$

We will use this to obtain a similar statement for sets in $\mathbb{Z}^{d}$ when one of the sets is sufficiently self-invariant, with counting measure replacing Lebesgue measure. Note that in general the re-statement of the Brunn-Minkowsky for $\mathbb{Z}^{d}$ is false. This is demonstrated by considering the "simplex" $A=B=\left\{0, e_{1}, \ldots, e_{d}\right\}$ with $e_{i}$ the standard generators of $\mathbb{Z}^{d}$.

We note that a variant of the Brunn-Minkowsky inequality for integer lattices was obtained by R.J Gardner and P. Gronchi in [2]. Also note that that I.Z. Ruzsa [6] has obtained inequalities for sums of sets in $\mathbb{Z}^{d}$ which are sufficient for the qualitative results stated below. However, the tight bound below does not follow from that work.

Denote by $e_{i} \in \mathbb{Z}^{d}$ the standard generators of $\mathbb{Z}^{d}$, that is, $\left(e_{i}\right)_{j}=\delta_{i, j}$.

Theorem 2.1. Let $A, B \subseteq \mathbb{Z}^{d}$ and $\delta>0$ and suppose that $A$ is $(1-\delta)$-invariant to $e_{1}, \ldots, e_{d}$, $i e$

$$
\left|A \cap\left(A+e_{i}\right)\right|>(1-\delta)|A|
$$

for all $i=1, \ldots, d$. Then

$$
|A+B| \geq\left(1-2 k^{2} \delta\right)\left(|A|^{1 / d}+|B|^{1 / d}\right)^{d}
$$

Proof. Let

$$
\begin{aligned}
A_{0} & =\left\{u \in A: u+e_{i} \in A \text { and } u+e_{i}+e_{j} \in A \text { for all } i, j=1, \ldots, d\right\} \\
& =A \cap \bigcap_{1 \leq i \leq d}\left(A-e_{i}\right) \cap \bigcap_{1 \leq i<j \leq d}\left(A-e_{j}-e_{i}\right)
\end{aligned}
$$

From the invariance assumption, $A \cap\left(A-e_{i}\right)$ and $A \cap\left(A-e_{i}-e_{j}\right)$ are of size at least $(1-2 \delta)|A|$, so

$$
\left|A_{0}\right|>\left(1-2 d^{2} \delta\right)|A|
$$


Consider now the sets $\widetilde{A}_{0}, \widetilde{B} \subseteq \mathbb{R}^{d}$ obtained by "thickening" $A_{0}, B$ :

$$
\widetilde{A}_{0}=A_{0}+[0,1)^{d}, \widetilde{B}=B+[0,1)^{d}
$$

(we identify $A_{0}, B$ with subsets of $\mathbb{R}^{d}$ in the obvious way). Clearly

$$
\nu\left(\widetilde{A}_{0}\right)=\left|A_{0}\right|, \nu(\widetilde{B})=|B|
$$

so by the Brunn-Minkowsky inequality and equation 2.1

$$
\begin{aligned}
\nu\left(\widetilde{A}_{0}+\widetilde{B}\right) & \geq\left(\left|A_{0}\right|^{1 / d}+|B|^{1 / d}\right)^{d} \\
& \geq\left(1-2 d^{2} \delta\right)\left(|A|^{1 / d}+|B|^{1 / d}\right)^{d}
\end{aligned}
$$

On the other hand, it is a simple consequence of the definition of $A_{0}$ that if $u \in \mathbb{Z}^{d} \cap\left(\widetilde{A}_{0}+\widetilde{B}\right)$ then $u \in A+B$, and therefore

$$
\widetilde{A}_{0}+\widetilde{B} \subseteq(A+B)+[0,1)^{d}
$$

so

$$
\nu\left(\widetilde{A}_{0}+\widetilde{B}\right) \leq|A+B|
$$

putting this all together, we get

$$
|A+B| \geq\left(1-2 d^{2} \delta\right)\left(|A|^{1 / d}+|B|^{1 / d}\right)^{d}
$$

One corollary of this is that in $\mathbb{Z}^{d}$, every Tempel'man sequence must have constant at least $2^{d}$.

\section{TEMPEL'MAN SEQUENCES AND ABELIAN RANK}

Suppose $G$ is a countable group and contains elements $e_{1}, \ldots, e_{d}$ which generate a subgroup isomorphic to $\mathbb{Z}^{d}$. Then theorem 2.1 can be extended to products of sufficiently invariant sets in $G$, where invariance is measured relative to $e_{1}, \ldots, e_{d}$.

We first assume that one of the sets is contained in this abelian subgroup:

Lemma 3.1. Let $G$ be a group and $H \cong \mathbb{Z}^{d}<G$. Let $A \subseteq H$ be $(1-\delta)$-invariant with respect to the standard generators $e_{1}, \ldots, e_{d} \in H$, ie $\left|\left(e_{i}+A\right) \cap A\right| \geq(1-\delta)|A|$ for $i=1, \ldots, d$. Then for any finite $B \subseteq G$,

$$
|B A|,|A B| \geq\left(1-2 d^{2} \delta\right)\left(|A|^{1 / d}+|B|^{1 / d}\right)^{d}
$$

Proof. We show the inequality for $|A B|$. Let $\left\{H g_{i}\right\}$ be an enumeration of the right cosets of $H$ and set $B_{i}=B \cap H g_{i}$. Using theorem 2.1,

$$
\begin{aligned}
|A B|= & \sum_{i}\left|A B_{i}\right| \\
& \geq\left(1-2 d^{2} \delta\right) \sum_{i}\left(|A|^{1 / d}+\left|B_{i}\right|^{1 / d}\right)^{d}
\end{aligned}
$$

using the fact that $t \mapsto\left(c+t^{1 / d}\right)^{d}$ is concave and homogeneous and that $\sum_{i}\left|B_{i}\right|=|B|$,

$$
\geq\left(1-2 d^{2} \delta\right)\left(|A|^{1 / d}+|B|^{1 / d}\right)^{d}
$$


Next we drop the requirement that the invariant set be contained in the abelian subgroup, and instead consider products of the form $F^{-1} F$ :

Lemma 3.2. Suppose $G$ is a group, $H \cong \mathbb{Z}^{d}<G$ with $e_{1}, \ldots, e_{d}$ the standard generators in $H$, and $F \subseteq G$ finite such that $F$ is $(1-\delta)$-invariant with respect to left multiplication by $e_{1}, \ldots, e_{d}$, ie $\frac{1}{|F|}\left|e_{i} F \cap F\right| \geq(1-\delta)|F|$. Then

$$
\left|F^{-1} F\right| \geq 2^{d}\left(1-2 d^{2} \sqrt{\delta}\right)(1-d \sqrt{\delta})|F|
$$

Proof. Let $H g_{i}$ be an enumeration of the right cosets of $H$ and $F_{i}=F \cap H g_{i}$. From the invariance assumption there is a set of indices $I$ such that

$$
\left|\cup_{i \in I} F_{i}\right| \geq(1-d \sqrt{\delta})|F|
$$

and $F_{i}$ is $(1-\sqrt{\delta})$-invariant to $e_{1}, \ldots, e_{d}$ from the left for each $i \in I$. Let $i_{0} \in I$ be such that $\left|F_{i_{0}}\right|$ is maximal among $\left\{\left|F_{i}\right|\right\}_{i \in I}$. Now

$$
\left|F^{-1} F\right| \geq\left|F_{i_{0}}^{-1} F\right| \geq \sum_{i \in I}\left|F_{i_{0}}^{-1} F_{i}\right| \geq
$$

by the lemma 3.1

$$
\geq \sum_{i \in I}\left(1-2 d^{2} \sqrt{\delta}\right)\left(\left|F_{i_{0}}\right|^{1 / d}+\left|F_{i}\right|^{1 / d}\right)^{d} \geq
$$

by choice of $i_{0}$

$$
\geq\left(1-2 d^{2} \sqrt{\delta}\right) \sum_{i \in I} 2^{d}\left|F_{i}\right| \geq 2^{d}\left(1-2 d^{2} \sqrt{\delta}\right)(1-d \sqrt{\delta})|F|
$$

Recall that the abelian rank $r(G)$ of $G$ is the largest $n$ such that $\mathbb{Z}^{d}$ can be embedded in $G$ (definition 1.2). Recall also the definition of Tempel'man sequences (definition 1.1). As an immediate corollary of lemma 3.2 we have

Theorem 3.3. If $r(G)<\infty$ then $2^{r(G)}$ is a lower bound on the constant associated with Tempel'man sequences in $G$.

For abelian groups, there is a converse to this, which together with theorem 3.5 characterizes those abelian groups which have Tempel'man sequences. I thank B. Weiss for suggesting the following construction:

Theorem 3.4. If $A$ is a countable abelian group with $r(A)<\infty$ then there are Tempel'man sequences in $A$.

Proof. Suppose that the rank of $A$ is $d<\infty$. We will construct a Tempel'man sequence. Let $\left\{a_{1}, a_{2}, \ldots\right\}$ be an enumeration of the elements of $A$. Let $A_{n}$ be the group generated by $\left\{a_{1}, \ldots, a_{n}\right\}$. Since $A_{n}$ is finitely generated, we can write it as $T_{n} \oplus \mathbb{Z}^{d(n)}$ with $T_{n}$ a finite group and $d(n) \leq d$. Set $F_{n}=T_{n} \times\{0, \ldots, k(n)\}^{d(n)}$, where $k(n)$ is chosen large enough that $F_{n}$ is $\frac{1}{n}$-invariant to $a_{1}, \ldots, a_{n}$ and $F_{n-1} \subseteq F_{n}$ (it is easy to check that such a $k(n)$ exists). Now one verifies that $\left|F_{n}-F_{n}\right| \leq 2^{d(n)}\left|F_{n}\right|$. Thus $\left\{F_{n}\right\}$ is a Tempel'man sequence.

From the proof one also sees that if $r(A)=d<\infty$ then there exist Tempel'man sequences with constant $2^{d}$, and lemma 3.2 shows that is the best possible. 
On the other hand, as noted in the introduction, finite rank in general does not guarantee the existence of Tempel'man sequences: the lamplighter group $L$ studied in 4 , has $r(L)=1$ but has no Tempel'man sequences.

From lemma 3.2 we also derive

Theorem 3.5. If $G$ is an amenable group with $r(G)=\infty$ then $G$ does not have Tempel'man sequences.

\section{Growth of tempered Sequences}

The previous section and the work of Lindenstrauss on the lamplighter group [4 show that there are amenable groups without Tempel'man sequences. However as noted in the introduction all amenable groups possess tempered sequences (definition 1.3), which can serve as averaging sequences. Ideally, we would like there to be averaging sequences which grow slowly (in $\mathbb{Z}$ they can grow linearly). It is therefore of interest to find conditions indicating or precluding such slow growth.

In order to analyze the growth rate of tempered sequences we will need information about the product of different sets, each of which is somewhat invariant:

Lemma 4.1. Suppose $G$ is a group, $H \cong \mathbb{Z}^{d}<G$ with $e_{1}, \ldots, e_{d}$ the standard generators in $H$, and $F_{1}, F_{2} \subseteq G$ finite such that $F_{1}, F_{2}$ are $(1-\delta)$-invariant with respect to left-multiplication by $e_{1}, \ldots, e_{d}$, ie $\left|e_{i} F_{k} \cap F_{k}\right| \geq(1-\delta)\left|F_{k}\right|$ for $i=1, \ldots, d$ and $k=1,2$. Then

$$
\left|F_{1}^{-1} F_{2}\right| \geq 2^{d}\left(1-2 d^{2} \sqrt{\delta}\right)(1-d \sqrt{\delta}) \min \left\{\left|F_{1}\right|,\left|F_{2}\right|\right\}
$$

Proof. Similar to lemma 3.2, Let $H g_{i}$ be an enumeration of the right cosets of $H$ and $F_{1, i}=$ $F_{1} \cap H g_{i}$ and similarly $F_{2, i}$. From the invariance assumption there are sets of indices $I, J$ such that

$$
\left|\cup_{i \in I} F_{1, i}\right| \geq(1-d \sqrt{\delta})\left|F_{1}\right|,\left|\cup_{j \in J} F_{2, j}\right| \geq(1-d \sqrt{\delta})\left|F_{2}\right|
$$

and $F_{k, i}$ is $(1-\sqrt{\delta})$-invariant with respect to left multiplication by $e_{1}, \ldots, e_{d}$ for each $i \in I$ and $k=1,2$. Let $i_{0} \in I$ be such that $\left|F_{1, i_{0}}\right|$ is maximal among $\left\{\left|F_{1, i}\right|\right\}_{i \in I}$ and $j_{0} \in J$ such that $\left|F_{2, j_{0}}\right|$ is maximal among $\left\{\left|F_{2, j}\right|\right\}_{j \in J}$. Now the calculation in lemma 3.2 shows that if $\left|F_{1, i_{0}}\right| \geq\left|F_{2, j_{0}}\right|$ then

$$
\left|F_{1}^{-1} F_{2}\right| \geq 2^{d}\left(1-2 d^{2} \sqrt{\delta}\right)(1-d \sqrt{\delta})\left|F_{2}\right|
$$

while if $\left|F_{2, j_{0}}\right| \geq\left|F_{1, i_{0}}\right|$ then

$$
\left|F_{1}^{-1} F_{2}\right| \geq 2^{d}\left(1-2 d^{2} \sqrt{\delta}\right)(1-d \sqrt{\delta})\left|F_{1}\right|
$$

which together complete the lemma.

Theorem 4.2. If $G$ is amenable and $r(G)=\infty$ then every tempered sequence in $G$ grows super-exponentially.

Proof. Let $\left\{F_{n}\right\}$ be a tempered Følner sequence in $G$, so for some constant $C,\left|F_{n-1}^{-1} F_{n}\right| \leq C\left|F_{n}\right|$ for all $n$. Fix $d>2+\log _{2} C$ and $\delta=\frac{1}{16 d^{2}}$. Let $H \cong \mathbb{Z}^{d}<G$ be generated by $e_{1}, \ldots, e_{d}$. For large enough $n$ the $F_{n}$ 's will be $(1-\delta)$-invariant to $e_{1}, \ldots, e_{d}$, so by lemma 4.1 for large enough 
$n$ we will have

$$
C\left|F_{n}\right| \geq\left|F_{n-1}^{-1} F_{n}\right| \geq 2^{d}\left(1-2 d^{2} \sqrt{\delta}\right)(1-d \sqrt{\delta}) \min \left\{\left|F_{n}\right|,\left|F_{n-1}\right|\right\}
$$

substituting $\delta=\frac{1}{16 d^{2}}$ and rearranging gives

$$
\left|F_{n}\right| \geq \frac{2^{d-2}}{C} \min \left\{\left|F_{n}\right|,\left|F_{n-1}\right|\right\}
$$

Since $d>2+\log _{2} C$, this relation excludes the possibility that $\min \left\{\left|F_{n-1}\right|,\left|F_{n}\right|\right\}=\left|F_{n}\right|$, so it must be that

$$
\left|F_{n}\right| \geq \frac{2^{d-2}}{C}\left|F_{n-1}\right|
$$

Since $d$ was arbitrary and this holds for all large enough $n$, the proof is complete.

The results presented in this paper do not settle the question of the character of averaging sequences in the class nonabelian groups of finite rank. In this class we have examples of groups with good sequences (eg nonabelian groups with polynomial growth) and groups with bad sequences (eg the lamplighter group $L$ ). Lindenstrauss has speculated that for finitely generated groups exponential growth may force super-exponential growth of tempered sequences the absence of Tempel'man sequences. The following example provides some further support for this. Note that unlike $L$, it is torsion-free.

Example. Let $\mathbb{Z}$ act on $\oplus_{i=-\infty}^{\infty} \mathbb{Z}$ by shift to the left, ie for $n \in \mathbb{Z}$ and $u=\left(u_{i}\right) \in \oplus_{i=-\infty}^{\infty} \mathbb{Z}$ we define $\left(u^{n}\right)_{i}=u_{i+n}$. Let

$$
G=\mathbb{Z} \ltimes \oplus_{i=-\infty}^{\infty} \mathbb{Z}
$$

explicitly, $G=\left\{(n, u) \in \mathbb{Z} \times \oplus_{i=-\infty}^{\infty} \mathbb{Z}\right\}$ and $(n, u) \cdot(m, v)=\left(n+m, u^{m}+v\right)$.

This group is finitely generated (eg. by $(0,(\ldots, 0,0,1,0,0, \ldots))$ and $(1,(\ldots, 0,0,0, \ldots))$ and is torsion-free. $G$ is also amenable, since it is solvable [3]. Clearly $r(G)=\infty$, so by theorem 4.2 $G$ has no Tempel'man sequences and every tempered sequence in $G$ grows super-exponentially.

\section{REFERENCES}

[1] R. J. Gardner. The Brunn-Minkowski inequality. bulletin of the American Mathematical Society, 39(3):355405, 2002.

[2] R. J. Gardner and P. Gronchi. A Brunn-Minkowski inequality for the integer lattice. Trans. Arer. Math. Soc., 353(10):3995-4024, 2001.

[3] Frederick P. Greenleaf. Invariant means on topological groups and their applications. Van Nostrand Reinhold Co., New York-Toronto, Ont.-London, 1969.

[4] Elon Lindenstrauss. Pointwise theorems for amenable groups. Inventiones Mathematicae, 146:259-295, June 2001.

[5] Donald Ornstein and Benjamin Weiss. The Shannon-McMillan-Breiman theorem for a class of amenable groups. Israel Journal of Mathematics, 44(1):53-61, 1983.

[6] I. Z. Ruzsa. Sum of sets in several dimensions. Combinatorica, 14(3):485-490, 1994.

[7] A. A. Tempel'man. Ergodic theorems for general dynamical systems. Dokl. Akad. Nauk SSSR, 176:790-793, 1967.

Current address: Einstein Institute of Mathematics, Edmond J. Safra Campus, Givat Ram, The Hebrew University of Jerusalem, Jerusalem 91904, Israel

E-mail address: mhochman@math.huji.ac.il 